\title{
ATIVIDADES INVESTIGATIVAS COM RECURSOS COMPUTACIONAIS NO ESTUDO DE SUCESSÕES NUMÉRICAS
}

\author{
Eleni Bisognin, Unifra, eleni@unifra.br \\ Maria do Carmo Barbosa Trevisan, Unifra, mcbtrevisan@ hotmail.com \\ Vanilde Bisognin, Unifra, vanilde@unifra.br
}

\begin{abstract}
Resumo
No presente trabalho descreve-se uma unidade didática, contendo atividades investigativas relacionadas com a variação da superfície lateral e o volume de um cubo, em função da aresta. Explora-se o conceito de sucessão numérica a partir da visualização gráfica de cubos com a aresta assumindo diferentes valores. Os cubos foram construídos com recursos computacionais, utilizados para subsidiar a preparação de professores de Matemática em relação ao uso pedagógico das Tecnologias da Informação e Comunicação - TICs. Trata-se de uma unidade didática, contendo atividades e animações para apoiar professores e alunos dos cursos de graduação tanto em relação ao uso de software computacional no ensino, como em relação à exploração de conceitos abstratos da Matemática. São descritas as atividades investigativas propostas, destacando-se o material produzido. Os resultados são parte de uma pesquisa desenvolvida com o propósito de analisar a eficácia de atividades investigativas associadas ao uso de recursos computacionais em sala de aula.
\end{abstract}

Palavras-chaves: investigação matemática; recursos computacionais; sucessão numérica.

\section{INVESTIGATIVE ACTIVITIES WITH COMPUTER RESOURCES IN THE STUDY OF NUMERICAL SEQUENCE}

\begin{abstract}
This paper describes a teaching unit containing investigative activities related to the variation of the lateral and the volume of a cube according to the edge. We explored the concept of numerical sequence from the graphical visualization of cubes with edge assuming different values, built with computational resources to support the preparation of teachers of mathematics in the educational use of Technologies of Information and Communication. This is a teaching unit with activities and animations to support teachers and students of graduate programs both in relation to the use of computer software in education, as for the exploration of abstract concepts of mathematics. We described the investigative activities with emphasis on the material produced. The results are part of a research conducted with the purpose of analyzing the effectiveness of investigative activities associated with the use of computational resources in the classroom.
\end{abstract}

Keywords : mathematics research, computational resources; numerical sequence.

\section{Introdução}

É consenso entre muitos professores que a Matemática, como ciência, e as Tecnologias da Informação e Comunicação - TICs estão cada vez mais presentes na vida das pessoas e na sociedade em geral. Por outro lado, à medida que a sociedade reconhece a importância da Matemática para a formação de cidadãos com habilidade de raciocínio lógico, rapidez, objetividade de sistematização, entre outras, tão necessárias 
para o mundo do trabalho atual; o que se observa, na escola, são altos índices de reprovação, evasão, bem como o fraco desempenho dos alunos em todos os níveis de ensino. Isso está constatado em exames oficiais, aplicados pelas Secretarias de Educação dos Estados e pelo Ministério de Educação.

Entre os muitos fatores que colaboram para esse cenário, encontra-se um fato ligado à prática dos docentes nas escolas, cujo trabalho não integra atividades relacionadas com a vida dos estudantes, tampouco elaboram atividades que envolvem o uso de tecnologias, disponíveis a todos os cidadãos. Isso contribui para que a escola seja um espaço com pouca motivação para a permanência e engajamento dos jovens. Assim, torna-se fundamentalmente importante a formação inicial e continuada de professores, para que possam implementar um tipo de ensino que vá ao encontro das aspirações e necessidades dos estudantes, uma vez que são as peças centrais do processo de ensino e aprendizagem.

A utilização de tecnologias da informação em suas diferentes formas, principalmente as de softwares computacionais, associadas a uma metodologia de ensino, pode trazer uma excelente colaboração para o processo de ensino e aprendizagem da Matemática na sala de aula, uma vez que facilitam a visualização gráfica, a descoberta de propriedades, a modelagem e a busca de resposta para problemas.

Acredita-se que as TICs, quando bem utilizadas, desempenham um papel essencial não só no processo de melhoria do ensino e aprendizagem da Matemática, mas também, tornam-se fatores facilitadores e motivadores para alunos e professores estudarem Matemática. Dessa forma, investir na formação inicial e continuada de professores, incluindo o preparo para o uso de recursos computacionais e considerando-se as dimensões técnica e pedagógica juntamente com uma reflexão crítica sobre o trabalho escolar desenvolvido, é imprescindível para que a melhoria da qualidade de ensino de fato aconteça.

Nos cursos de formação de professores, pensa-se que as TICs como recursos didáticos potencializam e favorecem o processo de ensino e aprendizagem da Matemática, pois são meios de comunicação e expressão, bem como fontes de informação, de conhecimento e suporte para um trabalho colaborativo. Dessa forma, a produção de materiais didáticos que requerem o uso de algum software computacional, associado a uma metodologia de ensino adequada, favorece não apenas o acesso às tecnologias, mas também possibilita que alunos e professores tenham condições de incorporá-los no fazer pedagógico.

Nesse contexto, no presente trabalho, descreve-se uma unidade didática em que se associou a metodologia de investigação matemática às ferramentas computacionais, para explorar os conceitos relacionados a sucessões numéricas, a partir da visualização gráfica da variação da área lateral de um cubo de aresta L e de volume V.

\section{Metodologia de investigação matemática}

Investigar significa formular questões cujas respostas não são conhecidas. De acordo com Ponte, Brocardo e Oliveira (2003, p.13) "investigar é procurar conhecer o que não se sabe". Portanto, uma atividade de investigação está sempre associada a um problema.

Como metodologia de ensino, a Investigação Matemática significa apresentar aos alunos propostas de trabalho que envolvam conceitos matemáticos, para que tenham a oportunidade de experimentar, discutir, formular, conjecturar, generalizar, provar, comunicar suas ideias e tomar decisões. 
Para Pereira (2004), o que diferencia a Investigação Matemática da Resolução de Problemas é que, nessa, a questão do problema é, de modo geral, bem definida, bem precisa e dada pelo professor, cujo objetivo é encontrar uma solução que não seja imediata. Na Investigação Matemática, o ponto de partida é aberto e cabe ao aluno e ao professor a formulação de questões, com a possibilidade de explorar diferentes caminhos a partir da mesma situação.

$\mathrm{Na}$ Resolução de Problemas é o professor quem gera a situação-problema e orienta todo o processo, deixando o método de resolução aberto para o aluno. Na Investigação Matemática, ao contrário, os alunos têm um papel mais ativo que o professor, o qual é apenas um moderador do trabalho de sala de aula. Essa metodologia exige do mestre a capacidade de reagir e interagir em situações improvisadas. Em aulas de Investigação Matemática, o professor deixa de ter o controle nas respostas dos alunos, nos métodos que eles utilizam e, em consequência disso, nos conteúdos que são tratados nas aulas, o que implica em uma mudança de atitude de sua parte.

A tabela a seguir, proposta por Ernest (1996) ilustra os papéis do professor e do aluno quando se trabalha a metodologia de Resolução de Problemas e de Investigação Matemática.

Tabela 1- Papel do professor e do aluno em diferentes abordagens.

\begin{tabular}{|l|l|l|}
\hline \multicolumn{1}{|c|}{ Metodologia } & \multicolumn{1}{|c|}{ Papel do professor } & \multicolumn{2}{|c|}{ Papel do aluno } \\
\hline Resolução de Problema & $\begin{array}{l}\text { Formula o problema e } \\
\text { deixa o método de de } \\
\text { resolução em aberto. }\end{array}$ & $\begin{array}{l}\text { Encontra seu própro } \\
\text { caminho para resolver o } \\
\text { problema. }\end{array}$ \\
\hline Investigação Matemática & $\begin{array}{l}\text { Escolhe uma situação de } \\
\text { partida (ou aprova a a a } \\
\text { escolha do aluno). }\end{array}$ & $\begin{array}{l}\text { Define os seus próprios } \\
\text { problemas dentro da } \\
\text { situação. Tenta resolver } \\
\text { pelo seu próprio caminho. }\end{array}$ \\
\hline
\end{tabular}

De acordo com Ponte, Brocardo e Oliveira (2003), o trabalho de uma Investigação Matemática na sala de aula envolve quatro momentos principais: o primeiro refere-se ao reconhecimento da situação, a sua exploração preliminar e a elaboração de questões; o segundo refere-se à formulação de conjecturas; o terceiro, à realização de testes e o refinamento de conjecturas; e o último, refere-se à argumentação, demonstração e avaliação do trabalho realizado. Esses momentos não são estanques e podem acontecer de modo simultâneo.

Esses momentos são descritos a seguir por meio da proposição de atividades relacionadas com o conceito de sucessão numérica, explorando a variação da área total e o volume de um cubo em função da aresta, com suporte computacional, que permite a visualização gráfica e auxilia na busca de respostas às questões formuladas.

\section{Unidade didática}

Nessa unidade são descritas atividades utilizadas com o propósito de construir o conceito de sucessão numérica e analisar seu comportamento. Isso foi feito por meio de recursos computacionais na elaboração de gráficos e representações que auxiliaram no desenvolvimento da tarefa.

Considere um cubo de aresta $1 \mathrm{~cm}$

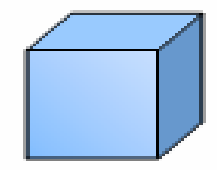


a) Qual é a área total do cubo? Qual é o volume do cubo?

b) Qual é a área total e o volume do cubo se a aresta for duplicada? O que você pode concluir em comparação com o resultado anterior?

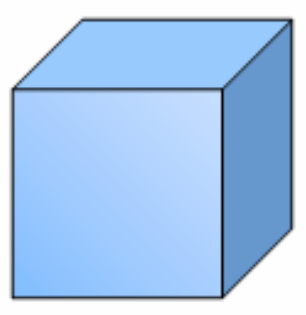

Cubo de aresta 2

c) Qual é a área total e o volume do cubo se a aresta for triplicada? O que se pode concluir comparando este resultado com os anteriores?

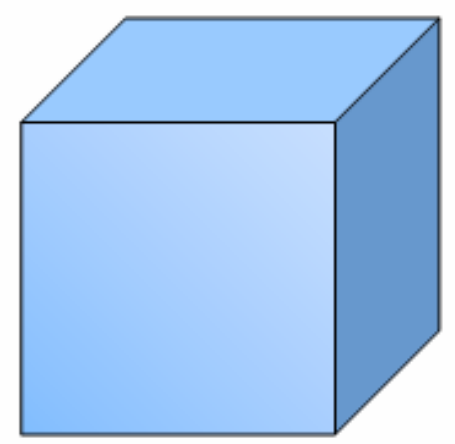

Cubo de aresta 3

d) Complete a tabela a seguir, escrevendo os valores da área total e do volume em função de outros valores da aresta.

Tabela2: valores da área total e do volume do cubo em função da aresta.

\begin{tabular}{|c|c|c|}
\hline Aresta $\mathbf{( c m )}$ & Área Total $(\mathbf{c m})^{\mathbf{2}}$ & ${\text { Volume }(\mathbf{c m})^{\mathbf{3}}}^{\mathbf{3}}$ \\
\hline 1 & $6 \times 1^{2}=6$ & $1^{3}=1$ \\
\hline 2 & $6 \times 2^{2}=24$ & $2^{3}=8$ \\
\hline 3 & $6 \times 3^{2}=54$ & $3^{3}=27$ \\
\hline 4 & $6 \times 4^{2}=96$ & $4^{3}=64$ \\
\hline 5 & $6 \times 5^{2}=150$ & $5^{3}=125$ \\
\hline 6 & $6 \times 6^{2}=216$ & $6^{3}=216$ \\
\hline 7 & $6 \times 7^{2}=294$ & $7^{3}=343$ \\
. & $\cdot$ &. \\
\hline
\end{tabular}




\begin{tabular}{|c|c|c|}
\hline & & \\
\hline 10 & $6 \times 10^{2}=600$ & $10^{3}=1000$ \\
\hline$\cdot$ & $\cdot$ & $\cdot$ \\
\hline
\end{tabular}

Analisando os valores da tabela, pode-se indagar:

e) À medida que a aresta aumenta, a área total e o volume do cubo também aumentam? Qual das duas medidas aumenta mais rapidamente?

f) O gráfico a seguir representa o crescimento da área total e do volume em função da aresta.

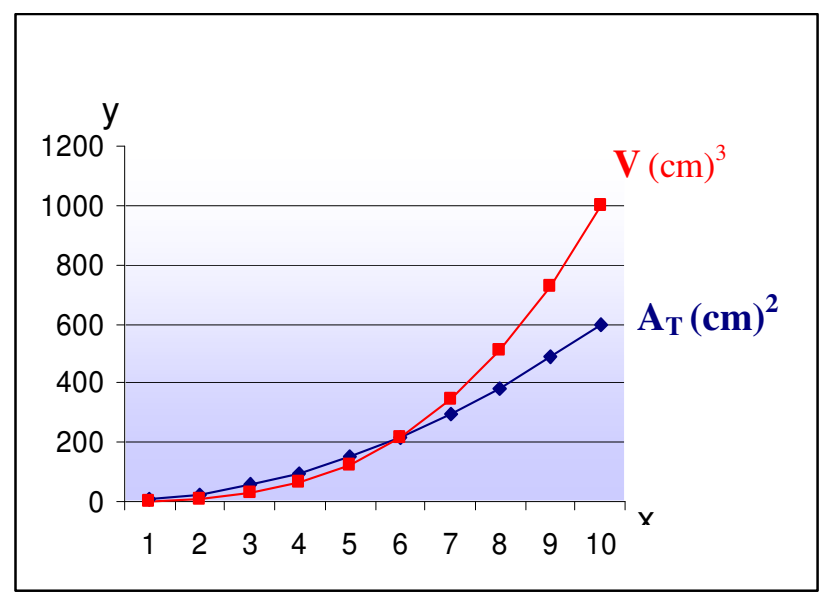

Gráfico 1: gráfico representativo da área total e do volume do cubo em função da aresta.

g) Analisando o gráfico, pergunta-se: para que valores da aresta a medida do volume é menor do que a medida da área total do cubo? E para que valores é maior? A área total e o volume do cubo crescem proporcionalmente ao crescimento da aresta?

A apresentação da atividade investigativa é uma etapa que pode influenciar no bom desempenho da tarefa. Para responder às questões propostas, isto é, para que se desenvolva o processo investigativo, é necessário compreender a situação, organizar os dados e fazer conjecturas que podem ser validadas ou refutadas.

h) Com os valores da tabela 2, construa uma nova tabela, estabelecendo a razão entre a área total A e o volume $\mathrm{V}$ do cubo. Os valores dessa razão formam uma sequência numérica. Analise e descreva o comportamento da sucessão obtida.

Tabela 3: Valores representativos da razão entre a área total e o volume do cubo. 


\begin{tabular}{|c|c|c|c|}
\hline Aresta $(\mathrm{cm})$ & Área Total $(\mathrm{cm})^{2}$ & Volume $(\mathrm{cm})^{3}$ & Razão: $\mathbf{A} / \mathbf{V}$ \\
\hline 1 & $6 \times 1=6$ & $1^{3}=1$ & $6 / 1=6$ \\
\hline 2 & $6 \times 4=24$ & $2^{3}=8$ & $24 / 8=3$ \\
\hline 3 & $6 \times 9=54$ & $3^{3}=27$ & $54 / 27=2$ \\
\hline 4 & $6 \times 16=96$ & $4^{3}=64$ & $96 / 54=1,5$ \\
\hline 5 & $6 \times 25=150$ & $5^{3}=125$ & $150 / 125=1,2$ \\
\hline 6 & $6 \times 36=216$ & $6^{3}=216$ & $216 / 216=1$ \\
\hline 7 & $6 \times 49=294$ & $7^{3}=343$ & $294 / 343=0,85$ \\
\hline . & . & & • \\
\hline . & . & & . \\
\hline 10 & $6 \times 100=600$ & $10^{3}=1000$ & $600 / 1000=0,60$ \\
\hline$\cdot$ & $\cdot$ & • & $\cdot$ \\
\hline$\cdot$ & . & $\cdot$ & · \\
\hline$\cdot$ & $\cdot$ & . & · \\
\hline
\end{tabular}

i) Que conjectura pode ser feita sobre o limite dessa sequência?

A análise do gráfico a seguir, que descreve o comportamento da sequência, auxilia na validação ou refutação da conjectura. Descreva suas conclusões.

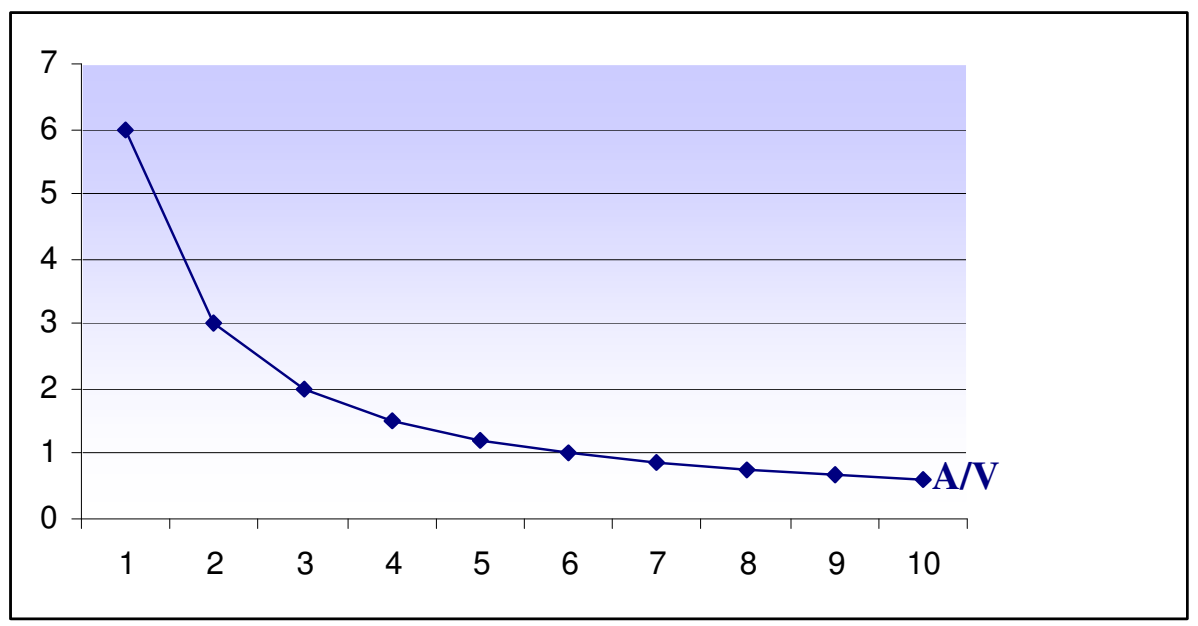

Gráfico 2: gráfico representativo dos valores obtidos pela razão entre a área total e o volume do cubo.

Outras questões importantes referem-se à análise da sequência construída, dividindose a aresta do cubo e mantendo seu o volume constante. Ao dividir-se a aresta do cubo original, novos cubos são construídos, fazendo com que a área total cresça em função da aresta, permanecendo o volume constante.

j) Se a medida $L$ da aresta do cubo é dividida ao meio, isto é, se $L=1 / 2$, quantos cubos de volume $V^{\prime}=(1 / 2)^{3}$ obtém-se de modo que o volume do cubo original, $V=1 \mathrm{~cm}^{3}$ permaneça constante? Observe a figura abaixo e analise o que acontece com a área total, considerando os novos cubos formados. 
k) Se a medida da aresta $\mathrm{L}$ é dividido por 3 , isto é, se $\mathrm{L}=1 / 3$, quantos cubos de volume $\mathrm{V}^{\prime}=(1 / 3)^{3}$ obtém-se para que o volume $\mathrm{V}=1 \mathrm{~cm}^{3}$ do cubo original permaneça constante? $\mathrm{O}$ que acontece com o valor da área total, neste caso? Analise a figura abaixo para comprovar sua conjectura.

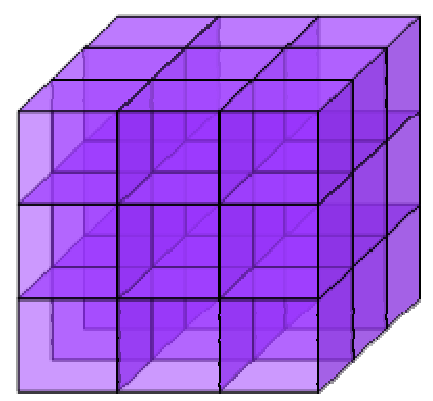

1) Continuando esse processo, o que acontece com a área total dos cubos formados se a aresta continuar a diminuir e o volume se manter constante? Que sequências numéricas são formadas? Complete a tabela a seguir até $n=10$ e analise seus comportamentos.

Tabela 4: número de cubos e área total em função da aresta

\begin{tabular}{|c|c|c|}
\hline Aresta $\mathbf{( c m )}$ & $\mathbf{N}^{\mathbf{0}}$ de cubos & Área Total $(\mathbf{c m})^{\mathbf{3}}$ \\
\hline 1 & 1 & 6 \\
\hline $1 / 2$ & 8 & 12 \\
\hline $1 / 3$ & 27 & 18 \\
\hline $1 / 4$ & 64 & 24 \\
\hline$\cdot$ & $\cdot$ & $\cdot$ \\
$\cdot$ & $\cdot$ & $\cdot$ \\
$\cdot$ & $\cdot$ & \\
& &
\end{tabular}

m) Observando os valores da tabela 4, qual é o número de cubos se a medida da aresta for dividida em $n$ partes iguais? O que acontece com a medida da área total? 
Ao completar a tabela 4, é possível observar que o volume do cubo original mantém-se constante, mas a área superficial cresce consideravelmente. Usando esse procedimento, é possível comparar os valores descritos em uma escala macro, com os valores descritos em uma escala micro e em uma nano escala. Esse é o princípio básico para a compreensão dos conceitos fundamentais da nanociência e da nanotecnologia.

\section{Considerações Finais}

A visualização gráfica desempenha um papel importante na formulação de conjecturas, que podem ser firmadas a seu respeito analiticamente. Assim,

visualizar não é o mesmo que ver. Em nosso contexto, visualizar é a habilidade para criar ricas imagens mentais que o indivíduo pode manipular em sua mente, promovendo diferentes representações do conceito e, se é necessário, usar o papel ou o computador para expressar a idéia matemática em questão(HITT, apud TORROBA et all, 2006, p.51).

Nesse trabalho revelaram-se as atividades de ensino, elaboradas por meio da metodologia da Investigação Matemática, associada a recursos computacionais, para explorar e construir conceitos matemáticos, com o propósito de subsidiar alunos e professores dos cursos de licenciatura em suas práticas de sala de aula. Acredita-se que a elaboração de um material pedagógico como aqui proposto, permite a transformação das práticas docentes dos sujeitos envolvidos. Sabe-se que não basta apenas tornar o uso do computador uma prática corriqueira mas, é preciso torná-lo uma ferramenta educacional de modo contribua efetivamente para a melhoria do processo de ensino e aprendizagem da Matemática.

O problema proposto pode ser resolvido, usando-se somente lápis e papel, sem o uso do computador. Porém, o uso de um software amplia as possibilidades de manipulações e de visualizações gráficas; favorece a exploração do conceito de sucessão numérica e a proposição de conjecturas, provocando a reflexão e o raciocínio matemático, bem como permite a formulação e resolução de novos problemas.

\section{5-Referências Bibliográficas}

ERNEST, P. Investigações, resolução de problemas e pedagogia. In.: ABRANTES, P.; LEAL, L. C.; PONTE, J. P. (Eds.). Investigar para aprender matemática. Lisboa, Portugal: Projecto MPT e APM, 1996, p. 25-48.

PONTE, J. P; BROCADO; J. E OLIVEIRA, H. Investigações matemáticas na sala de aula. Belo Horizonte: Autêntica, 2003.

PEREIRA, M.C. N. As investigações matemáticas no ensino-aprendizagem das sucessões: uma experiência com alunos do décimo segundo ano de escolaridade. Lisboa, Portugal: APM, 2004.

TORROBA,E.;REID.M.;RTCHEVERRY,N.;VILLAREAL,M. Los estudiantes proponem un problema:una possibilidad favorecida por los ambientes computacionales informatizados. In.: Revista Iberoamericana de Educación Matemática. México: , 2006,n 7, p.39-41. 
